\title{
ESTILO DE LIDERAZGO Y CREENCIAS ORGANIZACIONALES EN LA SATISFACCIÓN LABORAL DE LA ADMINISTRACIÓN PÚBLICA DE CHILE ${ }^{1}$
}

\author{
Autores: María Margarita Chiang Vega ${ }^{2}$ \\ Doctora en Recursos Humanos, Departamento de Administración y Auditoría, \\ Facultad de Ciencias Empresariales, Universidad del Bío-Bío, Concepción, Chile.

\begin{abstract}
Mauricio Salazar Botello
Magíster en Desarrollo y Comportamiento Organizacional, Departamento Gestión Empresarial, Facultad de Ciencias Empresariales, Universidad del Bío- Bío. Chillán, Chile.
\end{abstract}

Nelly M. Gómez Fuentealba ${ }^{4}$

Magíster en Estadística. Facultad de Ciencias, Universidad del Bío-Bío. Concepción, Chile.

\footnotetext{
${ }^{1}$ Proyecto de investigación DIUBB 115116 2/R Universidad del Bío-Bío, Concepción Chile

2 mchiang@ubiobio.cl

3 mauricio@ubiobio.cl

${ }^{4}$ ngomez@ubiobio.cl
} 
María José Martín Rodrigo 5

Doctora en Pedagogía. Departamento de Gestión Empresarial. Facultad de Ciencias Económicas y Empresariales. Universidad Pontificia Comillas, Madrid, España.

\title{
Resumen
}

Este estudio busca modelar el efecto que tiene en la satisfacción laboral, el estilo de liderazgo y las creencias organizacionales, contrastado en una muestra de 145 trabajadores de la Administración Pública de Chile. Para validar las hipótesis, se emplea una ecuación de regresión múltiple que arroja como resultado de una manera altamente significativa, que los estilos de creencias y liderazgo tienen una capacidad explicativa del 31\% de la satisfacción por el trabajo en general. Concretamente, los resultados señalan que el estilo de creencias de una cultura organizacional de control, influye negativa y significativamente sobre la satisfacción por el trabajo en general y que, a su vez, el estilo de liderazgo participativo, influye positiva y significativamente sobre la satisfacción en el trabajo en general.

Palabras Claves: satisfacción laboral, liderazgo, creencias organizacionales

How leadership styles and organizational beliefs affect Job satisfaction.

\begin{abstract}
This study aims to model the effect on job satisfaction leadership style and organizational beliefs in a universe made up of 145 workers. To contrast the hypotheses a multiple regression equation was applied which results shown that the beliefs and leadership styles have an explanatory power of $31 \%$ on the general job satisfaction which is highly significant. Specifically the style culture of control beliefs has a significantly negative influence on job satisfaction in general and the participative leadership styles a positive and significant influence on job satisfaction in general.
\end{abstract}

Key Words: job satisfaction, leadership, organizational beliefs.

\footnotetext{
${ }^{5}$ mariajo@cee.upcomillas.es
} 


\section{INTRODUCCIÓN}

En la actualidad, se requieren estilos de dirección diferentes, que estimulen nuevas relaciones internas en las organizaciones de trabajo. Para generar este cambio, es necesaria una forma distinta de ver el comportamiento de los individuos en las organizaciones que permita modificaciones de conductas individuales tendentes a dar cumplimiento a los requerimientos de la institución y a sus objetivos de negocio.

Esta investigación busca analizar en una muestra de organizaciones chilenas cómo, la satisfacción laboral, se ve afectada por el estilo de liderazgo y las creencias organizacionales.

\section{REVISIÓN DE LA LITERATURA}

Es fundamental la contribución de las disciplinas del Comportamiento Organizacional (Robbins, 1999) para comprender la conducta de las personas en el interior de la organización y es de mucha ayuda, a la hora de tratar de predecir o estimar algunos comportamientos en la misma.

Esto implica, de acuerdo con Hersey, Blanchard y Johnson (1998), que además de las habilidades técnicas, se necesitan también habilidades sociales para resolver los diferentes problemas organizacionales, siendo que la mayor parte de éstos, se encuentran en el nivel relacional de las personas y no a nivel de lo meramente material y administrativo. Por ello, para mejorar la gestión, parece importante estudiar el comportamiento organizacional.

\subsection{La satisfacción laboral ${ }^{6}$}

La creencia de que los empleados satisfechos son más productivos que los insatisfechos, ha sido una doctrina básica entre los empleadores durante años. Aunque existen muchas evidencias que ponen en duda esta supuesta relación causal, se puede argumentar que las sociedades avanzadas deben preocuparse no sólo por cuestiones materiales de la vida, es decir, por aspectos como una mayor productividad y adquisiciones materiales, sino también por su calidad. (Robbins, 1996).

La satisfacción laboral es importante en cualquier tipo de trabajo; no sólo en términos del bienestar deseable de las personas dondequiera que trabajen, sino también en términos de productividad y calidad. Así, la satisfacción es un tema de suma importancia para los empleados de cualquier empresa o institución, ya que la mayor parte de las personas pasan la tercera parte del día trabajando y lo

\footnotetext{
${ }^{6}$ Para mayor referencia véase Chiang y Ojeda (2013).
} 
hacen más de la mitad de su vida. La satisfacción laboral para la mayoría de los trabajadores constituye un fin en sí mismo o, un medio hacia el objetivo de la satisfacción personal.

La satisfacción y la insatisfacción laboral están asociadas con un gran número de indicadores organizacionales como la calidad del desempeño, bajas, rotación, rendimiento, etc. Por ello, la calidad de vida laboral hoy, es uno de los temas que ha captado mayor interés entre los investigadores de esta área.

Haciendo una revisión de la literatura del área de conocimiento en relación al concepto de satisfacción laboral, detectamos que este constructo ha sido definido de manera diferente por el elenco de autores que lo han abordado en sus investigaciones. (Harpaz, 1983, citado en Chiang, 2010).

La mayoría de las definiciones pueden agruparse en dos grandes bloques. Por un lado, se encuentran las que se refieren al tema como un estado emocional o afectivo (Crites, 1969; Locke, 1976; Price y Muller, 1986; Mueller y McCloskey, 1990; Muchinsky, 1993; citados en Berrios, Landa y Aguilar, 2006), siendo la de Locke (1976), la más original y conocida. Éste, plantea que la satisfacción laboral es un estado emocional positivo o placentero resultante de una percepción subjetiva de las experiencias laborales del sujeto. No se trata de una actitud específica, sino de una actitud general fruto de varias actitudes que un trabajador tiene hacia su trabajo y los factores con él relacionados.

Por otro lado, están las que la consideran como una actitud (Harpaz, 1983; Peiró, 1984; Griffin y Bateman, 1986; Bravo, Peiro y Rodríguez, 1996; Brief y Weiss, 2001; Citados en Chiang et al., 2010). Este segundo grupo de autores considera que la satisfacción laboral va más allá de las emociones y, de ahí la importancia que ésta tiene en las conductas laborales. Estos autores estudian la satisfacción laboral como una actitud generalizada ante el trabajo.

De este modo, entendemos pues la satisfacción laboral, como un concepto globalizador con el que se hace referencia a las actitudes de las personas hacia diversos aspectos de su trabajo. Por consiguiente, hablar de satisfacción laboral implica hablar de pensamientos, sentimientos y conductas. Así, Bravo, Peiró y Rodríguez, (1996) han definido satisfacción laboral como una actitud o conjunto de actitudes desarrolladas por la persona hacia su situación de trabajo, actitudes que pueden ir referidas hacia el trabajo en general o hacia facetas específicas del mismo.

\subsection{El liderazgo}

Koontz y O'Donnell (2000), afirman que el liderazgo consiste en influir en la gente para que se alineen en la consecución de cierta meta común. Según Kotter (1988), el liderazgo es el desarrollo de una visión, siendo capaz de lograr que los individuos apoyen determinadas estrategias y delegar poder en los mismos, para 
que se haga realidad dicha visión. A pesar de los obstáculos, Hersey y Blanchard (1972) dicen que el liderazgo es cualquier intento por influir en el comportamiento de un individuo o un grupo, en tanto que para McGregor (1991), el liderazgo es el proceso persuasivo o el modelo mediante el cual un individuo (o un grupo líder) induce a un grupo a perseguir los objetivos del líder o los objetivos compartidos por el mismo y sus seguidores. Dubrin (2000) dice que liderazgo es la influencia interpersonal orientada a lograr metas, y se alcanza a través de la comunicación. Estos autores concuerdan en que el liderazgo es el proceso de influir en las actividades de una persona o un grupo en los esfuerzos por alcanzar una meta en cierta situación. Es decir, hay liderazgo en cualquier situación en la que alguien trate de influir en el comportamiento de otro individuo o del grupo.

Luego se puede decir que, el proceso de liderazgo está en función del líder, el seguidor y otras variables situacionales. El liderazgo es la relación que se teje entre los líderes y sus seguidores. Es un proceso de influencia mutua, el cual genera un esfuerzo colectivo al servicio de los propósitos y valores comunes del líder y de sus seguidores. Cada vez que alguien intenta influir en el comportamiento de otro, aquel es el líder potencial y el sujeto de su influencia es el seguidor en potencia, sin importar si se trata del jefe, un colega, un subordinado, un amigo o bien todo un grupo.

En la teoría situacional Hersey y Blanchard (1972) indican que escoger el estilo correcto de liderazgo depende de la madurez de los seguidores, es decir del grado de preparación de la gente sobre la que el líder pretende ejercer su influencia. El concepto de madurez, definido por Hersey y Blanchard, se refiere a la medida en que las personas tienen la habilidad y la disposición de cumplir con una tarea específica. Una implicación interesante que se desprende de la teoría de Hersey y Blanchard, es la relación que se puede establecer entre la aplicación del estilo apropiado de liderazgo según la etapa de madurez de los seguidores, y la posibilidad de que éstos transiten hacia etapas de mayor madurez. Es decir, cuando el líder no se comporta según la madurez de sus seguidores, no sólo estaría atentando contra la eficiencia y la motivación de las personas, sino que también estaría impidiendo que los seguidores alcancen, en el futuro, mayores grados de madurez para la tarea. Bajo esta perspectiva, la adecuación del estilo de liderazgo a la madurez de los seguidores representa la posibilidad de ejercer un liderazgo que, en el tiempo, prepara a los seguidores para actuar de manera autónoma y auto-motivada.

\subsection{Creencias}

Las creencias se van formando a lo largo de nuestra vida. Constituyen la base sobre la cual se toman las decisiones, y se estructuran sobre los recuerdos. Esos recuerdos son un inventario de contenidos, pautas, interpretaciones, percepciones, etc.

Así como el funcionamiento de una organización estará determinado o definido por el conjunto de creencias, valores y actitudes que ésta posea ( ${ }^{\prime}$ Connor 
y Seymour, 1993), las creencias guían el comportamiento humano en sintonía con las necesidades del quehacer organizacional, delimitando y orientando las acciones operativas y sociales dentro de la organización. Estas creencias permiten que el trabajo que se realice dentro de la organización concuerde con su visión original, de la misma forma que éstas concuerden con el entorno.

Según Dilts (2004), las creencias se relacionan con las motivaciones más profundas que mueven a las personas a actuar o perseverar. En la medida que se conozcan las creencias que existen tras determinados comportamientos se podrá explicar en muchos casos los motivos por los cuales las personas se comportan de determinada manera. Si las personas creen firmemente que pueden hacer algo, lo harán, y si creen que algo es imposible, ningún liderazgo les convencerá de lo contrario. Cuando se cree ciegamente en algo, el individuo se comportará de un modo congruente con esa creencia.

$\mathrm{Al}$ analizar las distintas definiciones de los autores se desprende que las creencias son producto del aprendizaje de la organización y sus miembros. Estas definen las pautas de comportamiento dentro de una organización ya que llevan a las personas a interpretar los hechos de modo particular.

García y Dolan (1997) destacan la estrecha relación entre creencias y valores, estableciendo una secuencia de creencias y conductas, siendo las creencias la base de nuestras conductas, de tal manera que para modificar la conducta, más que modificar actitudes, lo que hay que hacer es modificar las creencias y valores. Entendemos pues por creencia, una serie de estructuras de pensamientos, elaboradas y arraigadas a lo largo del aprendizaje, que sirven para explicarnos la realidad y que preceden a la configuración de valores y conductas. Estos autores plantean que las creencias se agrupan en torno a dos culturas, la cultura del control y la cultura del desarrollo. La primera, - cultura del control -, se caracteriza por la lógica del pensamiento racional económico predominante desde inicios de la revolución industrial hasta finales del siglo XX. Ésta, tiende a basarse en controlar el rendimiento más que potenciarlo, reducir costes más que crear nuevas ideas e incluso, producir más que vender (Ostroff, 1999). La segunda, - cultura del desarrollo -, tiene sus inicios en la ya conocida investigación experimental en la Western Electric Company de Hawthorne (Hersey et al, 1998). La creencia sobre la que comienza a construirse es, que en la productividad de las personas inciden no sólo las variables materiales o económicas, sino que en el rendimiento, también influyen las variables de tipo social, como el sentirse perteneciente a un grupo, el estilo de liderazgo utilizado, los sistemas de reconocimiento no retributivo. Esto ratifica los resultados de la investigación realizada por los profesores Bonavía y Quintanilla (1999), en la que toman a McGregor, (1969), la teoría X, semejable a las creencias asociadas a la cultura del control y la teoría Y, semejable a las creencias de la cultura del desarrollo

Concluyendo, la inclinación hacia algunas de estas culturas configura el comportamiento directivo que imperará en las instituciones, ya sea con una orien- 
tación fuerte a la participación, desarrollo, creatividad, trabajo en equipo, en el caso de la cultura del desarrollo, o con una orientación jerarquizada, controladora, poco participativa, en el caso de la cultura del control.

\subsection{Satisfacción laboral, Liderazgo y Creencias}

La relación entre Satisfacción laboral, Liderazgo y Creencias es un tema que ha despertado nuestro interés y como tal, se constituye en el objeto de estudio de esta investigación. Hasta ahora, los aspectos más estudiados en la literatura, han sido las relaciones que se establecen entre la conducta de liderazgo tradicional y el desarrollo de la satisfacción. En general, se han encontrado correlaciones positivas y significativas entre la conducta de liderazgo, tales como la iniciación de estructura y la consideración, y la satisfacción laboral. Lo podemos constatar en Cuadra y Veloso (2007), donde se observa que el liderazgo tiene una correlación positiva estadísticamente significativa con la satisfacción laboral en general. También se ha encontrado relación entre la conducta de recompensa del líder y la satisfacción laboral (Podsakoff, Todor, y Grover, 1984). Sin embargo no se ha encontrado resultados que relacionen los tres conceptos en una ecuación donde estaría la satisfacción laboral (como variable dependiente) siendo afectada por el estilo de liderazgo y por las creencias organizacionales. Es nuestra intención arrojar luz con esta investigación sobre este aspecto.

\section{METODOLOGÍA}

Para lograr el objetivo de la investigación, se desarrolla un estudio empírico transversal, descriptivo y correlacional.

\subsection{Descripción de la muestra}

El universo escogido para esta investigación lo constituyen trabajadores de organizaciones públicas chilenas. La selección de este campo de estudio obedece a la necesidad de aportar información relevante que pueda favorecer a una mejor gestión de las instituciones, puesto que en la actualidad la gestión es un parámetro importante para el análisis de la calidad del servicio prestado a sus clientes. Se seleccionó una muestra aleatoria de 145 trabajadores, 91 (63\%) mujeres y 54 (37\%) hombres.

\subsection{Aplicación del instrumento}

Tras la socialización inicial y la explicación del procedimiento a los sujetos de la muestra, los datos se recogieron aplicando un cuestionario de auto respuesta, 
anónima y se aplicó sin alterar el funcionamiento normal de las actividades cotidianas.

a) Parte I. Cuestionario de Satisfacción Laboral desarrollado y validado por Chiang, Salazar, Huerta y Núñez (2008), para medir la satisfacción laboral en trabajadores. La escala utilizada consta de 11 ítems. Los trabajadores responden utilizando un formato de respuesta tipo Likert de cinco puntos.

b) Parte II. Cuestionario de Liderazgo. Para medir los estilos de liderazgo se utilizó un cuestionario de 36 ítems (ver Cuadro $\mathrm{N}^{\circ} 3$ ) desarrollado por Chiang, Gómez y Salazar (2011).

c) Parte III. Cuestionario de Creencias Organizacionales. Para medir creencias organizacionales se utilizó el cuestionario desarrollado y validado por Salazar y Chiang, (2007). Este cuestionario consiste en 28 ítems, con dos escalas de 14 ítems cada una (Cuadro $\left.\mathrm{N}^{\circ} 4\right)$.

En las partes I y II del instrumento, los trabajadores responden utilizando un formato de respuesta de Likert de cinco puntos (de "Totalmente de acuerdo = 5" a "Totalmente en desacuerdo $=1$ "). Las valoraciones de las escalas se obtienen mediante el promedio de los valores de los elementos de cada escala.

\subsection{Análisis de los datos}

Una vez obtenidos los datos fue necesario organizarlos, clasificarlos y resumirlos adecuadamente, de tal manera que se posibilitara un mejor análisis de la información obtenida. Para tabular los datos se utilizó una hoja de cálculo Excel, luego se utilizó el paquete estadístico SPSS 13.0, mediante el que se obtuvieron los resultados descriptivos y las relaciones existentes entre las variables estudiadas.

\section{RESULTADOS Y DISCUSIÓN}

\subsection{Análisis de fiabilidad (coeficiente de consistencia interna o indicador de unidimensionalidad)}

Por fiabilidad se entiende lo que indican los coeficientes de consistencia interna, es decir, el coeficiente Alpha de Cronbach. Lo que se quiere comprobar es si cada ítem mide lo mismo y si las respuestas tienden a covariar, es decir, si los sujetos responden de una manera coherente y de esta manera, se pueda deducir que los ítems expresan el mismo rasgo. Schmitt (1996) señala que es usual considerar un valor de coeficiente de fiabilidad de 0,70 como aceptable. 
Sin embargo en caso de variables "blandas", como el liderazgo y la satisfacción laboral, se acepta 0,6.

El Cuadro 1 muestra que las estimaciones de la consistencia interna de las escalas de creencias arrojan una fiabilidad adecuada para cada uno de los conceptos estudiados, es decir las escalas diferencian adecuadamente unos grupos de trabajo de otros.

\section{Cuadro 1. Análisis de fiabilidad de las escalas de liderazgo: Alfa de Cronbach}

\begin{tabular}{|c|l|c|c|}
\hline & & Privado & Público \\
\hline I & Satisfacción laboral & 0,91 & 0,88 \\
\hline II & Estilos de Liderazgo & entre 0,56 y 0,91 & entre 0,72 y 0,89 \\
\hline III & Creencias organizacionales & entre 0,71 y 0,72 & 0,75 \\
\hline
\end{tabular}

Fuente: elaboración propia.

\subsection{Hipótesis de la investigación}

$\mathrm{H}_{1 \mathrm{~A}}$ : Las creencias de la cultura del control tienen un impacto sobre la satisfacción por el trabajo en general.

$\mathrm{H}_{1 \mathrm{~B}}$ : Las creencias de la cultura del desarrollo tienen un impacto sobre la satisfacción por el trabajo en general

$\mathrm{H}_{2 \mathrm{~A}}$ : El estilo de liderazgo directivo tiene un impacto sobre la satisfacción por el trabajo en general.

$\mathrm{H}_{2 \mathrm{~B}}$ : El estilo de liderazgo persuasivo tiene un impacto sobre la satisfacción por el trabajo en general.

$\mathrm{H}_{2 \mathrm{C}}$ : El estilo de liderazgo participativo tiene un impacto sobre la satisfacción por el trabajo en general.

$\mathrm{H}_{2 \mathrm{D}}$ : El estilo de liderazgo de delegación tiene un impacto sobre la satisfacción por el trabajo en general.

\subsection{Resultados}

Para contrastar las hipótesis de la investigación se emplean la siguiente ecuación de regresión múltiple:

\section{$S T G=\beta_{0}+\beta_{1} C C C+\beta_{2} C C D+\beta_{3} L D I+\beta_{4} L P E+\beta_{5} L P A+\beta_{6} L D E+e$}

Dónde:

La variable dependiente STG = Satisfacción por el trabajo en general 
Y las variables independientes son:

$\mathrm{CCC}=$ Creencias cultura del control

$\mathrm{CCD}=$ Creencias cultura del desarrollo

LDI $=$ Liderazgo Directivo o instructivo

LPE $=$ Liderazgo Persuasión

LPA $=$ Liderazgo Participación

LDE $=$ Liderazgo Delegación

Los resultados de los principales estadísticos descriptivos se observan en el Cuadro 2:

\section{Cuadro 2. Estadísticos descriptivos}

\begin{tabular}{|l|c|c|c|}
\hline \multicolumn{1}{|c|}{ Variables } & Media & $\begin{array}{c}\text { Desviación } \\
\text { estándar }\end{array}$ & Muestra \\
\hline Satisfacción por el trabajo en general & 3,80 & 0,71 & 170 \\
\hline Creencias cultura del control & 2,49 & 0,58 & 170 \\
\hline Creencias cultura del desarrollo & 4,04 & 0,47 & 170 \\
\hline Liderazgo Directivo o instructivo & 3,47 & 0,76 & 170 \\
\hline Liderazgo Persuasión & 3,47 & 0,82 & 170 \\
\hline Liderazgo Participación & 3,45 & 0,81 & 170 \\
\hline Liderazgo Delegación & 3,02 & 0,58 & 170 \\
\hline
\end{tabular}

Fuente: elaboración propia.

Estos resultados ilustran que los niveles de satisfacción por el trabajo en general de la muestra consultada alcanza una puntuación de 3,80 sobre un máximo de 5 , pudiendo ser calificados como niveles más que regulares. Mientras que los niveles de creencias se pueden clasificar sólo como regulares (2,49 para CCC y 4,04 para CCD, sobre un máximo de 5). En cuanto a los factores de liderazgo también se puede observar que en general éstos alcanzan niveles regulares $(3,47$ LDI y LPE, 3,45 LPA y 3,02 LDE). 
Cuadro 3. Correlaciones de Pearson

\begin{tabular}{|l|c|c|c|c|c|c|c|}
\hline \multicolumn{1}{|c|}{ Variables } & CCC & CCD & LDI & LPE & LPA & LDE & STG \\
\hline Creencias cultura del control & 1 & & & & & & \\
\hline Creencias cultura del desarrollo & $-0,12$ & 1 & & & & & \\
\hline Liderazgo Directivo & 0,08 & $0,26 * *$ & 1 & & & & \\
\hline Liderazgo Persuasión & 0,03 & $0,18 * *$ & $0,64 * *$ & 1 & & & \\
\hline Liderazgo Participación & 0,01 & 0,22 & $0,62 * *$ & $0,81 * *$ & 1 & & \\
\hline Liderazgo Delegación & $0,26 * *$ & $0,19 * *$ & $0,38^{* *}$ & $0,32 * *$ & $0,29 * *$ & 1 & \\
\hline Satisfacción por el trabajo en general & $-0,19 * *$ & $0,18^{* *}$ & $0,32 * *$ & $0,46 * *$ & $0,49 * *$ & 0,09 & 1 \\
\hline
\end{tabular}

** Significativo al $1 \%$

Fuente: elaboración propia.

En el Cuadro 3 se presentan las correlaciones de Pearson. Estos resultados indican que la satisfacción por el trabajo en general correlaciona de manera positiva y significativa con las creencias cultura del desarrollo (CCD), liderazgo directivo, persuasivo y participativo, y de manera negativa y significativa con las creencias cultura del control (CCC).

Seguidamente en el Cuadro 4 se presenta la capacidad explicativa del modelo empleado para explicar la satisfacción a partir de las variables de creencias y liderazgo.

\section{Cuadro 4. Capacidad explicativa del modelo}

\begin{tabular}{|l|c|c|c|c|}
\hline \multicolumn{1}{|c|}{ Modelo } & R & R cuadrado & Test F & Sig. \\
\hline 1 & 0,55 & 0,31 & 12,00 & 0,00 \\
\hline
\end{tabular}

Fuente: elaboración propia.

Como se puede observar a partir del Cuadro 4 la capacidad explicativa de los estilos de creencias y liderazgo sobre la satisfacción por el trabajo en general es del $31 \%$.

$\mathrm{El}$ test $\mathrm{F}$ asociado al análisis de varianza destaca de manera estadísticamente significativa (Sig. $<0,01$ ), por lo cual se concluye que al menos una de las variables explicativas del modelo, influye en la satisfacción por el trabajo en general. 
Finalmente en el Cuadro 5 se presenta la incidencia estadística de cada uno de los estilos de creencias y liderazgo.

\section{Cuadro 5. Análisis de coeficientes}

\begin{tabular}{|l|c|c|c|c|c|}
\hline \multicolumn{1}{|c|}{ Modelo } & $\begin{array}{c}\text { Coeficiente no } \\
\text { estandarizado }\end{array}$ & $\begin{array}{c}\text { Error } \\
\text { estándar }\end{array}$ & $\begin{array}{c}\text { Coeficiente } \\
\text { estandarizado }\end{array}$ & Test t & Sig. \\
\hline Constante & 2,30 & 0,48 & & 4,77 & $0,00 * *$ \\
\hline Creencias cultura del control & $-0,19$ & 0,09 & $-0,15$ & $-2,19$ & $0,03 *$ \\
\hline Creencias cultura del desarrollo & 0,09 & 0,10 & 0,06 & 0,85 & 0,40 n.s. \\
\hline Liderazgo Directivo & 0,05 & 0,08 & 0,05 & 0,55 & 0,58 n.s. \\
\hline Liderazgo Persuasión & 0,15 & 0,11 & 0,17 & 1,39 & 0,17 n.s. \\
\hline Liderazgo Participación & 0,30 & 0,11 & 0,34 & 2,76 & $0,01^{*}$ \\
\hline Liderazgo Delegación & $-0,04$ & 0,09 & $-0,04$ & $-0,47$ & 0,64 n.s. \\
\hline
\end{tabular}

Fuente: elaboración propia.

\section{CONCLUSIONES}

Los resultados nos permiten establecer las siguientes conclusiones:

1. Los estilos de creencias y liderazgo tienen una capacidad explicativa del $31 \%$ de la satisfacción por el trabajo en general, altamente significativa desde el punto de vista estadístico en estos temas.

2. El estilo de creencias "cultura del control" influye negativa y significativamente sobre la satisfacción por el trabajo en general (Test $\mathrm{t}=-2,19$; Sig. $=$ 0.03 ). Es decir a mayor presencia de la "cultura del control" disminuye la satisfacción laboral de los trabajadores, les disminuye el gusto por su trabajo cuando aumenta el control en sus labores diarias y disminuye la libertad para crear (Chiang, 2008).

3. El estilo de liderazgo participativo influye positiva y significativamente sobre la satisfacción por el trabajo en general (Test $\mathrm{t}=2,76$; Sig. $=0,01$ ). Es decir a mayor presencia del estilo de liderazgo participativo aumenta la satisfacción laboral. En otras palabras, a estos trabajadores les gusta participar en las decisiones, sentir que aportan en las decisiones que luego deben aplicar en sus trabajos.

Desde esta perceptiva, se observa que en casi todas las situaciones, las personas se inclinan por las creencias y estilos que estimulen el desarrollo, 
la participación, el compromiso, la formación para el desarrollo y en general todo aquello que implique un bienestar para las personas. Esto lleva a pensar que todo aquello que está relacionado con tendencias que estimulan el control, el cumplimiento de objetivos, el cumplimiento de normas, entre otras, no es aceptado por los profesionales de todos los niveles. Casi todos, al momento de ser entrevistados o hablar con ellos informalmente, rechazaban las prácticas basadas en medidas de control. Sin embargo, en el momento de someterlos a un análisis más sistemático, como contestar un cuestionario, surgen inclinaciones claras hacia los estilos Directivos, los cuales privilegian las medidas de control para la ejecución o desarrollo de las tareas. Esto puede indicar que aún no estamos preparados para asumir en la práctica un estilo más de desarrollo o que, en definitiva, se contempla la importancia de éste, pero no se está preparado para implementarlo en la organización, como líder o como seguidor.

\section{BIBLIOGRAFÍA}

BERRIOS-MARTOS, M. P., AUGUSTO-LANDA, J. M., y AGUILARLUZON, M. C. (2006), "Inteligencia emocional percibida y satisfacción laboral en contextos hospitalarios. Un estudio exploratorio con profesionales de enfermería", Index de Enfermería, núm.54, 30-34.

BONAVIA, T. y QUINTANILLA, I. (1999), "Creencias directivas y participación de los empleados", Revista de psicología del trabajo y de las organizaciones., núm.15, (3), 367-383.

BRAVO, M., PEIRÓ, J. y RODRÍGUEZ, I. (1996), "Satisfacción laboral”. En J. Peiró y F. Prieto (Eds.). Tratado de psicología del trabajo, 1. La actividad laboral en su contexto (pp. 343-394), Madrid: Ed. Síntesis S.A.

BRIEF, A. P. y WEISS, H. M. (2001), "The affective dimensions of organizational behavior”, Working paper, New Orleans, Estados Unidos; Tulane University.

CHIANG, M., SALAZAR, M., HUERTA, P. y NÚÑEZ, A. (2008), “Clima organizacional y satisfacción laboral en organizaciones del sector estatal (Instituciones Públicas).Desarrollo, adaptación y validación de instrumentos", Revista Universum, Universidad de Talca., núm 3, (23), 67-86.

CHIANG, M., MARTÍN, M. J., y NÚÑ̃Z A. (2010), Relaciones entre el Clima Organizacional y la Satisfacción Laboral, Madrid: Publicaciones de la Universidad Pontificia Comillas.

CHIANG, M., GÓMEZ, N. y SALAZAR, M. (2011), "La relación existente entre las creencias organizacionales y el estilo de liderazgo, en una institución pública de educación. Desarrollo y validación de un instrumento", 
Ponencia en el XXVII Encuentro Nacional de Facultades de Administración y Economía: ENEFA, Chile: Universidad del Bío Bío.

CRITES, J. O. (1969), The study of vocational behavior and development. Vocational Psychology. Nueva York: McGraw-Hill.

CUADRA, A. y VELOSO, C. (2007), "Liderazgo, clima y satisfacción laboral en las organizaciones", Revista Universia, núm 2 (22), 42-58.

DILTS, R. (2004), Coaching, herramientas para el cambio, Barcelona: Ediciones Urano.

DUBRIN, A. J. (2000), Fundamentos de administración. $5^{\text {a }}$ ed., México: Thomson.

GARCIA, S. y DOLAN, S. (1997), Dirección por Valores. Madrid: Ediciones McGraw-Hill.

GRIFFIN, R. W. y BATEMAN, T. S. (1986), "Job Satisfaction and Organizational Commitment". En C.L. Cooper e I. Robertson (Eds), International Review Industrial and Organization Psychology (pp. 157-188), Nueva York: John Wiley \& Sons.

HARPAZ, I. (1983), Job satisfaction: Theoretical perspectives and a longitudinal analysis. New York: Libra Publishers.

HERSEY, P. \& BLANCHARD, R. (1972), Management of organizational behavior, Nueva Jersey: Ed. Prentice Hall.

HERSEY, P., BLANCHARD, K. y JOHNSON, D. (1998), Administración del Comportamiento Organizacional, liderazgo situacional, New Jersey: Ed. Prentice Hall.

KOTTER, J. P. (1988), The leadership factor, New York: Free Press.

KOONTZ, H. y O'DONNELL, C. (2000), Administration general, New Jersey: Medicines Prentice Hall.

LOCKE, E. A. (1976), "The nature and causes of job satisfaction". En M.D. Dunnette (Ed.), Handbook of Industrial and Organizational Psychology, Chicago: Rand McNally.

MCGREGOR, D. (1969), El aspecto humano de la empresa, México: Ediciones Diana.

MUCHINSKY, P. M. (1993), Psychology applied to work $4^{\circ}$ ed., California: Pacific Grove Publishing Company.

MUELLER, C. W. y MCCLOSKEY, J. C. (1990), "Nurse's job satisfaction. A proposed measure", Nursing Research, núm.39, 113-117.

O'CONNOR, J. y SEYMOUR, J. (1993), Introducción al PNL, Barcelona: Ediciones Urano.

OSTROFF, F. (1999), La organización horizontal, Oxford: Oxford University Press.

PEIRÓ, J. M. (1984), Psicología de la Organización. $2^{\circ}$ Ed., Madrid: UNED, Ministerio de Educación y Ciencia. 
PODSAKOFF, P. M., TODOR, W. D., GROVER, R. A. y HUBER, V. L. (1984), "Situational moderators of leader reward and punishment behaviors: Fact or fiction?", Organizational Behavior and Human Performance, núm. 34(1), 1-63.

PRICE. J. L. y MUELLER, C. W. (1986), Handbook of Organizational Measurement. Marshfield, MA: Pittman.

ROBBINS, S. (1999), Comportamiento Organizacional, México: Ediciones Prentice Hall. Hispanoamericana,

SALAZAR, C. y CHIANG, M. (2007), "Creencias organizacionales, desarrollo y validación de un instrumento", Revista ICADE. núm.72, 75-92. 


\section{ANEXO. CUESTIONARIOS}

\section{CREENCIAS ORGANIZACIONALES}

\section{CREENCIAS}

1 No tener tiempo para la familia y uno misma es signo de éxito en la vida.

2 Las personas cambian su comportamiento si sienten que trabajan en un ambiente agradable, desafiante y justo.

3 Para las personas el cambio es una oportunidad, no una amenaza

4 Las personas se preocupan de cumplir las normas y reglas más que de solucionar los problemas

5 Las relaciones en el trabajo deben ser impersonales y regidas por criterios formales

6 Tratar a las personas con educación y respeto es esencial para promover compromisos y el

buen funcionamiento en el lugar de trabajo.

7 Los sentimientos personales no deben interferir de ninguna manera en los objetivos de la organización

8 Las personas están motivadas básicamente por incentivos económicos y siempre actuarán

buscando mayores beneficios económicos.

9 Las personas en la institución son el factor clave para que ocurran los cambios

10 La satisfacción de necesidades sociales y la realización de sí mismo lleva a la persona a cumplir los objetivos de la institución.

11 Las personas cambian su comportamiento cuando el cambio está de acuerdo con lo que creen, piensan y sienten

12 Para las personas por lo general es más importante hablar que escuchar

13 Las personas persiguen su beneficio personal a expensas de los demás

14 Las personas están abiertas a trabajar en los nuevos desafíos del entorno y de la organización

15 Los recursos de capital son más valiosos que los recursos humanos

16 El interés por las personas genera más gastos que beneficios

${ }_{17}$ Cuando las personas son tratadas como responsables, autónomas y creativas, es probable que terminen siéndolo

18 A las personas en el trabajo no se les paga para pensar.

19 La mayor parte de las personas tienen que ser dirigidas, controladas y obligadas a trabajar

20 Las personas cambian su comportamiento si sienten que son reconocidas y esto es una oportunidad de desarrollo para todos

21 Las personas buscan constantemente asumir más responsabilidades

22 Las personas por lo general comparten los valores definidos por la organización y se compro-

22 meten de acuerdo a ellos.

23 En el trabajo la responsabilidad es de las personas y no del equipo.

24 Se necesitan personas preparadas con capacidades intelectuales y emocionales para cumplir con el trabajo

25 Cuando las personas cometen errores hay que buscar al culpable 


\section{CREENCIAS}

26 Los problemas y errores detectados a cualquier nivel deben ser confrontados y analizados abiertamente

27 El trabajo en equipo es difícil y consume demasiado tiempo

28 La imaginación, el ingenio y la creatividad es una característica de la mayoría de las personas en la institución 


\section{LIDERAZGO}

\section{LIDERAZGO}

1 Mis directivos insisten en el empleo de procedimientos uniformes y en la necesidad de cumplir las tareas

2 Mis directivos hablan con los trabajadores y luego determinan los objetivos

3 Cuando mis directivos inician una interacción amistosa con un trabajador, le explicita que 3 debe ser consciente de su rol

4 Mis directivos hacen participar a los trabajadores para tratar de resolver los problemas juntos

5 Mis directivos incorporan las recomendaciones de los trabajadores pero dirigen el cambio

6 Mis directivos permiten la participación de los trabajadores en la determinación de metas, pero no los apremia

7 Mis directivos hacen lo posible para que los trabajadores se sientan importantes y comprometidos

8 Mis directivos estimulan a los trabajadores a trabajar sobre los problemas y permite que se discutan

9 Mis directivos son cautos y no intervienen

10 Mis directivos discuten el desempeño previo con los trabajadores y luego analizan la necesi-

10 dad de prácticas nuevas

11 Mis directivos permiten la participación de los trabajadores en la toma de decisiones y apoyan 11 los aportes valiosos

12 Mis directivos enfatizan la importancia de las tareas y plazos

13 Mis directivos hacen lo posible para que los trabajadores se sientan importantes y comprometidos

14 Mis directivos discuten la situación con los trabajadores y luego inician los cambios necesarios

15 Mis directivos permiten la participación de los trabajadores para elaborar el cambio, pero no los apremia

16 Mis directivos dejan que los trabajadores trabajen en libertad

17 Mis directivos hacen participar a los trabajadores en la forma de decisiones, pero controlan que se cumplan las metas

18 Mis directivos ensayan la solución con los trabajadores y analizan la necesidad de prácticas nuevas

19 Mis directivos no perjudican las relaciones con sus colaboradores por ser demasiado directas

20 Mis directivos dejan la solución en manos de los trabajadores

21 Mis directivos buscan la aprobación del cambio por parte de los trabajadores y les ceden la 21 implementación

22 Mis directivos intencionalmente no intervienen

23 Mis directivos actúan con rapidez y firmeza para corregir y volver a dirigir

24 Mis directivos no toman ninguna medida determinante

25 Mis directivos permiten la participación del equipo en la redefinición de los objetivos, pero no los apremia 


\section{LIDERAZGO}

26 Mis directivos permiten a los trabajadores seguir su rumbo

27 Mis directivos incorporan las sugerencias de los trabajadores, pero controlan que los objetivos 27 sean cumplidos

28 Mis directivos redefinen los estándares y supervisan cuidadosamente

29 Mis directivos dejan que los trabajadores seleccionen los medios que les parezcan adecuados 29 para alcanzar las metas de su área

30 Mis directivos definen el cambio y supervisan cuidadosamente

31 Mis directivos anuncian los cambios y luego los implantan supervisando de cerca

32 Mis directivos permiten a los integrantes de los trabajadores buscar la solución por si mismos

33 Mis directivos encaminan a los trabajadores a trabajar de una manera bien definida

34 Mis directivos definen las metas y supervisan cuidadosamente

35 Mis directivos evitan las confrontaciones sin ejercer presión

36 Mis directivos actúan con rigidez y firmeza para corregir y volver a dirigir 
Ma Margarita Chiang, Mauricio Salazar, Nelly N. Gómez y Mª José Martín

\section{SATISFACCIÓN LABORAL}

\begin{tabular}{|r|l|}
\hline & SATISFACCIÓN \\
\hline 1 & La relación con sus colegas \\
\hline 2 & El apoyo directivo que usted recibe \\
\hline 3 & Con respecto a la libertad que se le otorga para elegir su propio metodología de trabajo \\
\hline 4 & En mi trabajo circula la información \\
\hline 5 & En mi trabajo hay espíritu de colaboración y ayuda \\
\hline 6 & Hay buena relación entre los miembros de la empresa \\
\hline 7 & La jefatura me estimula para mejorar mi trabajo \\
\hline 8 & Reconocimiento que se obtiene por un buen trabajo \\
\hline 9 & Atención que se presta a sus sugerencias \\
\hline 10 & La autonomía que tiene para planificar su labor \\
\hline 11 & Su participación en las decisiones de su área o departamento \\
\hline
\end{tabular}

